\title{
2,4,6-tribromophenol inhibit overall thyroid function in mouse
}

\author{
Dinh Nam Tran, Dongoh Lee, Song Ai Kang, Jin Yong An and Eui-Bae Jeung \\ Laboratory of Veterinary Biochemistry and Molecular Biology, Chungbuk National University, \\ Cheongju, Chungbuk, 28644, Republic of Korea
}

\section{EP586: Endocrine disruptors}

\section{ABSTRACT}

Synthesized chemicals that not existed in the environment have been troubled endocrine physiology. These endocrine disruptors (EDs) had been noted and replaced with other chemicals. Most of compound banned for production act as an estrogen. But other than estrogen, especially thyroid hormone related EDs are noted for early brain and neural development. Brominated flame retardants (BFRs) are representative known as thyroid disruptor because of its structural similarity. 2,4,6-tribromophenol (TBP), one of BFRs, has been produced bulky for reagent of thermostable plastic. Its effect to thyroid system related with transthyretin (TTR) is studied in vivo. TTR is blood protein that has a high binding affinity to thyroid hormones. ICR female mouse, post-natal 16 days, were exposed to TBP with or without triiodothyronine (T3) or thyroxine (T4). Liver and pituitary gland were dissected and RNA was extracted by Trizol. mRNA level of each pituitary gland and liver was quantified using quantitative real time PCR. Serum level of each free thyroid hormone was measured by Immulite 1000. Dio1 was increased in pituitary gland and liver by TBP. Reduction of thyroid hormone receptor beta, isoform 2 (ThrB2) and Dio2 inductions of Gh and thyroid stimulating hormone $B$ (TshB) were observed in pituitary gland. Thyroid hormone receptor beta, isoform 1 (ThrB1) was reduced by TBP in liver. Each free thyroid hormone level was decreased by TBP and its inhibition effect was also observed when treatment with thyroid hormones. TBP was shown anti-thyroid effect in some way such as Dio1, Dio2, TshB and ThrB2 in pituitary gland. TBP inhibited expression of Dio1 and ThrB1 induced by thyroid hormones in liver. Finally, TBP decrease the hormone level in blood. Like other BFRs, TBP also shown interfering effect to thyroid system.

\section{RESULT}

\section{BFR}

pesticide partition Coefficien

$\log$ Kow $=4.13$

Water solubility $70 \mathrm{mg} / \mathrm{L}\left(\right.$ at $17^{\circ} \mathrm{C}$ )

antifungal agent intermediate

Molecular Weight $330[\mathrm{~g} / \mathrm{mol}]$

Figure 1. Products that contain TBP. Chemical structure and properties of TBP. Modified from Deng J. (2010).

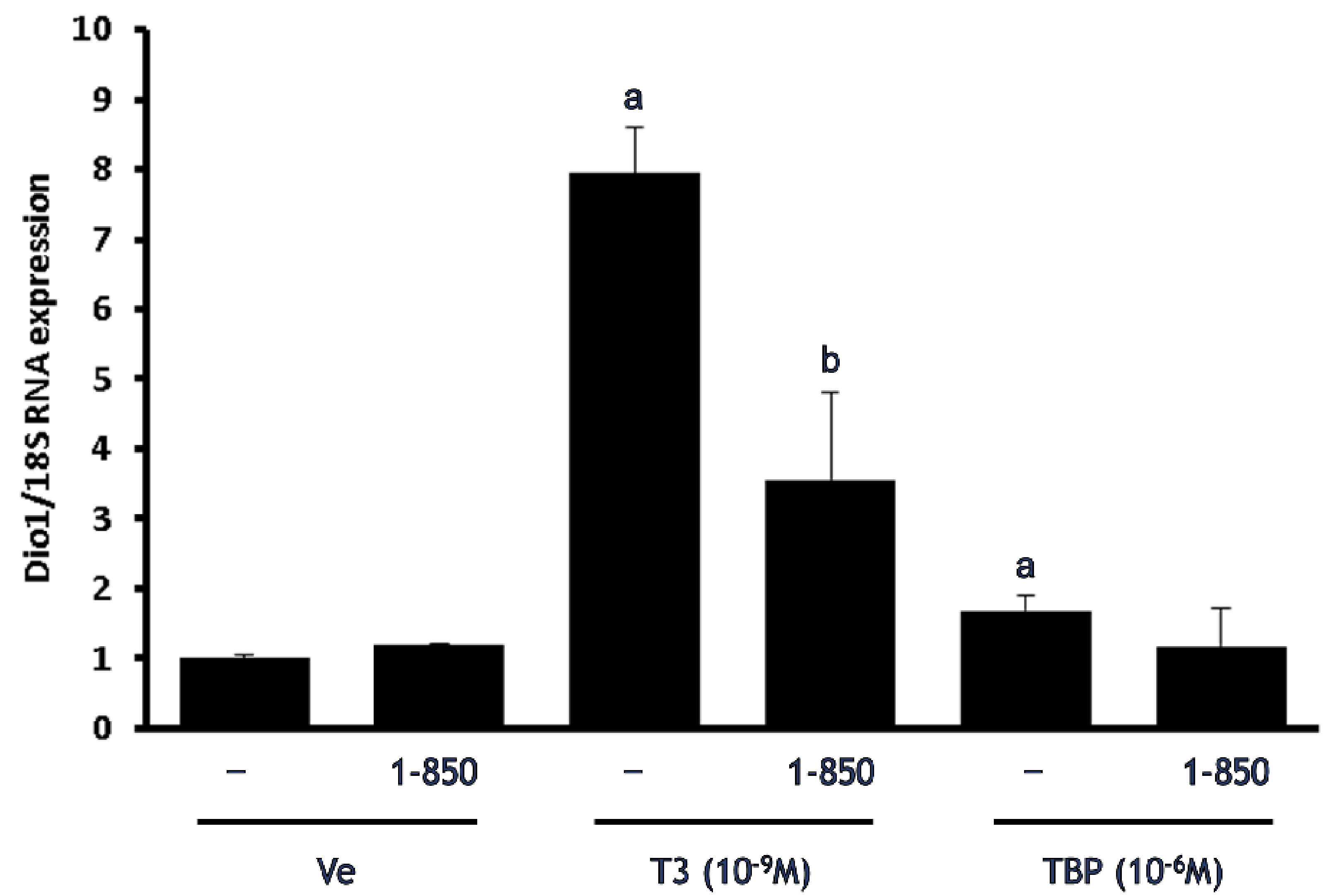

Figure 2. Relative mRNA expression level of TBP on GH3 cell. Bar graph is divided major in chemicals and subdivided with or without thyroid receptor antagonist 1-850 (shown as 1-850). Chemicals is shown as Ve; vehicle, T3; triiodothyronine, TBP; 2,4,6-tribromophenol. Each P-value present, a $P<0.05$ versus vehicle, ${ }^{\mathrm{b} P}<0.05$ versus without $1-850$. Data are presented as mean \pm SD.

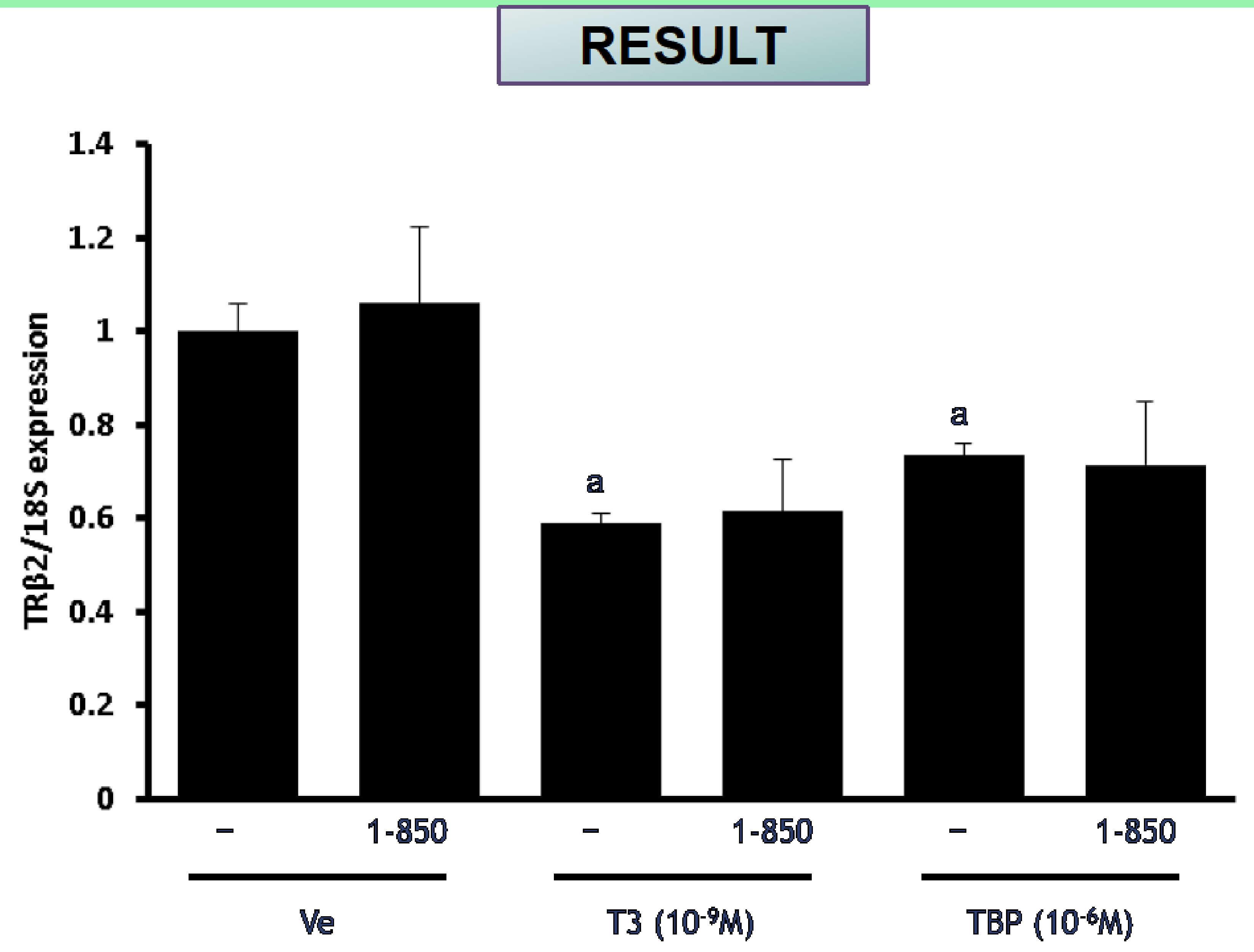

Figure 3. Relative mRNA expression level of thyroid receptor $B$ isoform 2 on GH3 cell. Bar graph is divided major in chemicals and subdivided with or without thyroid receptor antagonist $1-850$ (shown as 1-850). Chemicals is shown as Ve; vehicle, T3; triiodothyronine, TBP; 2,4,6-tribromophenol. Each $P$-value present, ${ }^{a} P<0.05$ versus vehicle, ${ }^{b} P<0.05$ versus without $1-850$. Data are presented as mean \pm SD

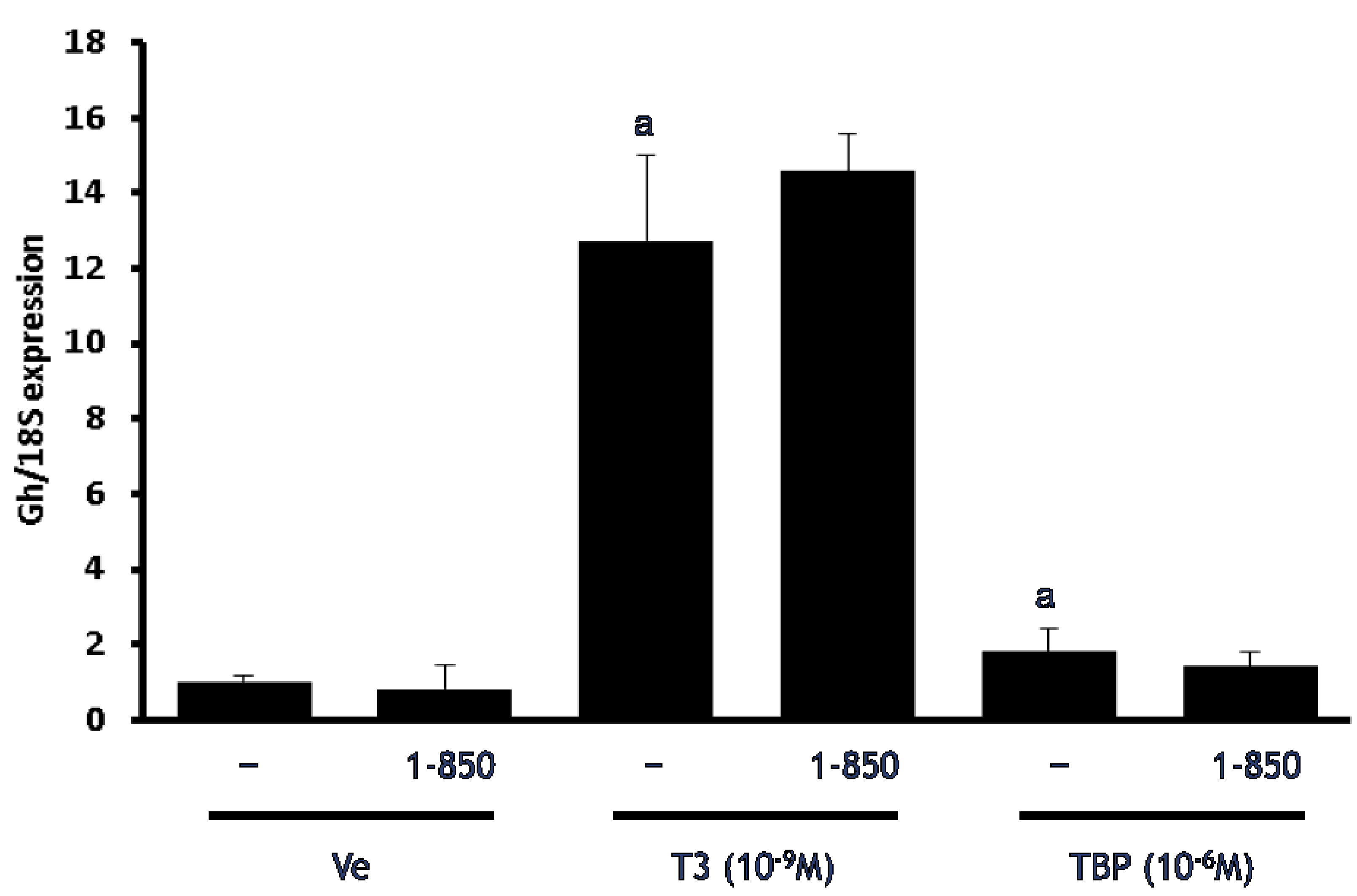

Figure 4. Relative mRNA expression level of growth hormone on GH3 cell. Bar graph is divided major in chemicals and subdivided with or without thyroid receptor antagonist 1-850 (shown as 1-850). Chemicals is shown as Ve; vehicle, T3; triiodothyronine, TBP; 2,4,6-tribromophenol. Each P-value present, ${ }^{\mathrm{a} P} \mathrm{P}<0.05$ versus vehicle, ${ }^{\mathrm{b}} \mathrm{P}<0.05$ versus without $1-850$. Data are presented as mean \pm SD

\section{CONCLUSION}

Dio1 was up-regulated by T3 at 8 fold and TBP at 1.8 fold. Thyroid receptor antagonist 1-850 decreased expression of Dio1 at T3. Dio1 was also decreased at TBP, but not significantly. This supported that TBP induced the Dio1 mediated by thyroid receptor. Thyroid receptor beta 2 expression was decreased at T3 and TBP, but not altered in 1850. ThrB is divided into 2 of isoform that ThrB2 is majorly expressed in hypothalamus and pituitary gland. This result shows that induction of Dio1 is not mediated by ThrB2. Induction of growth hormone was increased at T3 and TBP, but its expression was not decreased by 1850. Though thyromimetic effect of TBP is not quite immense, we should be warned considering its environmental universe and lipoophilic properties.

\section{REFFERENSE}

Deng J., Liu C., Yu L., Zhou. (2009). Chronic exposure to environmental levels of tribromopheno impairs zebrafish reproduction. Toxicol Appl Pharmacol._243(1):87-95

Covaci A., Harrad S., Abdallah M.A., Ali N., Law R.J., Herzke D., de Wit C.A.(2011). "Novel brominated flame retardants: a review of their analysis, environmental fate and behaviour. Environ Int. 37(2):532-56 National Center for Biotechnology Information. PubChem Compound Database; CID=1483, https: //pubchem.ncbi.nlm.nih.gov/compound/1483?from=summary\#section=Top 